\title{
Exactitude d'intonation et précision de l'ensemble dans la musique de l'Australie centrale
}

Catherine J. Ellis

Traducteur : Isabelle Schulte-Tenckhoff

\section{OpenEdition}

Journals

Édition électronique

URL : http://journals.openedition.org/ethnomusicologie/1592

ISSN : 2235-7688

Éditeur

ADEM - Ateliers d'ethnomusicologie

Édition imprimée

Date de publication : 1 octobre 1991

Pagination : 207-226

ISBN : 978-2-8257-0437-8

ISSN : 1662-372X

Référence électronique

Catherine J. Ellis, « Exactitude d'intonation et précision de l'ensemble dans la musique de l'Australie centrale », Cahiers d'ethnomusicologie [En ligne], 4 | 1991, mis en ligne le 01 janvier 2012, consulté le 19 avril 2019. URL : http://journals.openedition.org/ethnomusicologie/1592 


\title{
EXACTITUDE D'INTONATION \\ ET PRÉCISION DE L'ENSEMBLE \\ DANS LA MUSIQUE \\ DE L'AUSTRALIE CENTRALE*
}

\author{
Catherine J. Ellis
}

Cet article vise à démontrer l'habileté et la maîtrise d'exécution propres à la tradition vocale non accompagnée d'Australie centrale. Il s'intègre à un projet de recherche plus étendu mené par L.M. Barwick et moi-même sur vingt-six interprétations d'une série majeure de chants, enregistrées par différents chercheurs au cours d'une cinquantaine d'années. Les chants en question proviennent de plusieurs groupes régionaux distincts qui appartiennent au seul «major song area» du peuple de langue Pitjantjatjara. Ces données importantes, assemblées par Barwick à partir de sources multiples, fournissent de précieuses informations sur les structures sous-tendant cette tradition, et c'est à la lumière de ces données que seront exposées celles retenues dans ces pages. En elle-même, l'analyse quantitative entreprise ici a déjà fourni quelques renseignements importants, alors qu'elle ne porte que sur deux séries - respectivement désignées par les sigles KE et KK - que j'ai enregistrées sur le terrain avec beaucoup de soin pour assurer notamment une reproduction exacte de la hauteur des sons. Conjointement avec d'autres analyses structurelles, l'étude en question fait apparaître une tradition vocale qui exige une connaissance et un savoir-faire considérables chez le meneur du chant collectif, ainsi que chez les membres du groupe qui ont pour tâche de chanter à l'unisson avec le meneur désigné selon la tradition. Dans ses travaux antérieurs, Barwick (voir 1989 ; 1990) a su démontrer la signification d'un «enclenchement» minutieux du texte et de la mélodie. Sur la base d'une série encore différente, j'ai essayé de mettre en évidence un processus d'imbrication semblable (Ellis 1985) ; et plus récemment (Ellis s.d.), j'ai tenté un exposé des éléments essentiels de la structure tonale des "petits chants ${ }^{1}$ de cette série particulière.

Un trait saillant de toute pratique musicale en Australie centrale est la présence de signaux donnés en cours d'exécution, qui maintiennent l'unité fonctionnelle de l'ensemble; et dans bien des cas, la nature des erreurs commises durant l'exécution est révélée par l'examen des signaux prêtant à confusion au

Traduit de l'anglais par Isabelle Schulte-Tenckhoff.

Terme par lequel les interprètes différencient l'élément d'une série de chants de la série ellemême. 
moment du chant. Ainsi le présent article attire-t-il l'attention sur quelques exemples où un désaccord entre chanteurs devient audible, afin de montrer comment une mauvaise exécution vocale par certains affecte la structure globale du chant. Une place de choix reviendra à la pratique vocale d'ensemble, notamment à sa mise en œuvre par rapport à la structure tonale du chant interprété; deux aspects sous-jacents seront également abordés, à savoir le rapport entre texte et mélodie et le chant à l'unisson comme idéal culturel.

\section{Les éléments de l'ensemble}

En comparant diverses exécutions d'un même chant, Barwick (1990) a démontré que, si le texte et la mélodie sont relativement fixes, leurs combinaisons possibles sont fort variées. En recherchant les principes qui gouvernent l'enclenchement des deux, elle décrit la manière dont divers types de refrains sont adaptés au contenu mélodique unificateur de tel ou tel segment du chant ${ }^{2}$. Les refrains repérés dans diverses analyses comprennent les plus usuels: deux vers répétés - A et $\mathrm{B}$ - constituent la base du modèle isorythmique entier (dans le texte en question, c'est $\mathrm{AABB}$ ). Notons que A ne vient pas toujours en premier; il y a un processus d'inversion des vers qui se répercute sur l'emplacement des paroles et de leur rythme syllabique correspondant par rapport à la formule mélodique. Barwick discute des «jointures» textuelles entre refrains dans le texte répété cycliquement, c'est-à-dire entre $\mathrm{AA}$ et $\mathrm{BB}$, entre $\mathrm{BB}$ et AA. Ces jointures larges coïncident avec des segments mélodiques majeurs (voir aussi Ellis 1985: 106ss, 217-21). Il existe de nombreuses autres jointures plus restreintes dans les segments et les cellules rythmiques, qui seront toutefois laissées de côté ici.

Le meneur commence seul chaque «petit chant», communiquant ainsi au groupe le texte qui va être chanté, la position du texte par rapport à la mélodie, le cadre rythmique du texte et la hauteur sélectionnée. En ce qui concerne cette dernière, de petits intervalles (toujours avec un écart de $6 \mathrm{~Hz}$ environ à travers tout le chant) sont utilisés pour signaler le début d'une nouvelle série d'intervalles. Lorsque le groupe risque de se tromper, le meneur tend à les entonner vigoureusement.

La notion d'idéal que représente l'unisson est suggérée par les expressions uwankara inkanyi («chacun chante») et tjunguringanyi («se rassemblant»). Toutefois, le discours tenu par les interprètes sur l'acte communautaire du chant ne fait jamais ressortir très clairement ce que l'unisson signifie en termes structurels. Il apparaît que, dans certaines régions, on encourage l'harmonie, en particulier dans la ligne mélodique descendante retardée exécutée par quel-

Selon les interprètes, la mélodie du chant représente le «parfum» des ancêtres; le chant est considéré, à son tour, comme le lien principal entre l'interprète et la terre. 
ques chanteurs dans la partie centrale du chant. Mais j'ai cru comprendre en suivant des interprétations vocales sur le terrain, que dans la région de langue pitjantjatjara, avec laquelle je suis la plus familière, l'importance accordée à tjunguringanyi exprime les avantages du chant à l'unisson. Les interprètes avec lesquels j'ai travaillé décrivent parfois le fait de chanter en harmonie comme un «bruit». Le chant en octaves existe également, mais il est généralement considéré comme un exploit particulier. Les chanteurs traditionnels pitjantjatjara qui enseignaient aux étudiants de musique blancs à l'Université d'Adelaïde se sont finalement aperçus que les difficultés plus grandes rencontrées par les femmes par comparaison avec les hommes tenaient au fait qu'elles devaient entonner à l'octave supérieure. C'est grâce à la présence d'une chanteuse que les étudiantes ont pu résoudre leur problème. La résonance provoquée par le chant à l'unisson favorise en quelque sorte l'intonation correcte, et cette résonance n'est pas aussi apparente dans le chant octavié.

Nombreux sont les ouvrages qui abordent la question du chant à l'unisson ${ }^{3}$, montrant que l'unisson est soit le mode, soit un mode préféré de chant. Ils affirment également que deux ou plusieurs parties se déroulent simultanément lorsque certains membres du groupe se rapprochent ou s'éloignent de la tonique avant les autres. J'ai moi-même décrit la surimposition apparemment aléatoire du même petit chant lorsqu'il est exécuté à la fois par les chanteurs et par les danseurs qui appliquent leurs peintures corporelles en se tenant éloignés des chanteurs. Cela provoque, tantôt une exécution simultanée à des hauteurs différentes, tantôt un chant antiphonique. Dans une telle situation, lorsqu'il s'agit de meubler le temps nécessaire à l'application des peintures élaborées sur le corps, l'important est d'interpréter correctement le petit chant, et non de le faire à l'unisson (voir Ellis 1984).

La conception du chant à l'unisson et octavié diffère d'une région à l'autre. Jones (1980: 712) note que dans certaines régions, on rencontre la polyphonie, qui prend la forme d'un bourdon, d'octaves, de tierces, de quartes ou de quintes parallèles, d'un canon, ou encore de deux parties différentes exécutées indépendamment. La plupart de ces développements du chant à l'unisson se retrouvent dans les régions septentrionales où l'on joue aussi du didjeridu ${ }^{4}$. Il n'en demeure pas moins vrai que, dans la musique considérée ici, c'est l'unisson qui est recherché.

Afin de préserver l'ensemble, les interprètes doivent être en mesure de recourir à leur propre expérience de la série de chants, de suivre les signaux donnés par le meneur et de chanter à l'unisson. Les chanteurs confirmés se distinguent des autres par leur capacité de pénétrer davantage la signification du texte fixe et du rythme syllabique correspondant, plutôt que par l'apprentissage de combinaisons toujours plus élaborées entre les deux. C'est l'emboîtement

Voir notamment Strehlow (1971), McCardell (1976), Ellis et al. (1978), Moyle (1979; 1984), Wild (1984) et Tunstill (1985).

4 Didjeridu : aérophone fait d'un tube en bois, long d'un mètre et demi environ [n.d.l.r.]. 
entre texte et mélodie qui requiert le plus d'habileté d'exécution de la part de l'interprète, car le rapport entre les deux change constamment en vertu de facteurs inhérents au processus d'interprétation lui-même. L'interdépendance texte/mélodie ne peut être envisagée que comme un processus, alors que le rapport texte/rythme est donné et se prête donc à être mémorisé. En ce qui concerne l'intonation correcte, l'état présent de la recherche fait apparaître l'existence d'un système de hauteurs que les chanteurs aborigènes tiennent pour fondamental: il faut le respecter rigoureusement pour réaliser un bon ensemble ${ }^{5}$.

La méthode de quantification suivie pour énumérer ces tons a déjà été décrite en détail (voir Ellis 1965). Elle offre divers avantages, bien qu'il existe maintenant des procédés plus sophistiqués pour chiffrer la hauteur des chants collectifs. En effet, elle mesure la durée pour chaque fréquence, indiquant donc automatiquement les notes le plus souvent utilisées dans chaque exécution d'un petit chant. En revanche, elle n'articule pas spécifiquement le texte et la hauteur - articulation qui serait fort précieuse pour la recherche en cours. Néanmoins, les exemples dont il sera question mettent clairement en évidence ce qui se passe lorsqu'il y a erreur.

Les travaux les plus récents sur ces données (voir Ellis s.d.) démontrent que les tons principaux de la première mélodie de cette série de chants, bien que, apparemment, distants de la tonique d'une quinte $(3: 2)$ et d'une quarte (4:3), présentent une marge d'erreur plus petite lorsqu'on les considère comme étant séparés par les différences de fréquence de $108 \mathrm{~Hz}, 72 \mathrm{~Hz}, 54 \mathrm{~Hz}$ et $36 \mathrm{~Hz}$. De plus, les interprétations où la quarte tend à être accentuée plus que la quinte se servent d'une tonique qui place la quarte à la même différence de fréquence que la quinte dans d'autres exécutions de la «même» mélodie. Ainsi une exécution organisée autour d'une tonique de $108 \mathrm{~Hz}$ comporte l'intervalle de $3: 2$ à $162 \mathrm{~Hz}$ (c'est-à-dire une différence de fréquence de $54 \mathrm{~Hz}$ entre la tonique et la quinte). Une grande partie de la série KE attribue cette hauteur à la tonique. En revanche, la série KK utilise fréquemment une tonique à $158 \mathrm{~Hz}$, et la principale note aiguë est à $212 \mathrm{~Hz}$, ce qui équivaut à une différence de fréquence de $54 \mathrm{~Hz}$ et à un rapport de presque 4:3 (210.6 étant exactement à 4:3 au-dessus de la tonique). D'autre part, les mesures déterminées pour la série KK indiquent que la transposition dans le petit chant se fait seulement au moyen de la différence de fréquence. Pourtant, dans des interprétations différentes, il est évident que les exécutants utilisent un de ces deux procédés de transposition possibles, car certains d'entre eux transposent l'intervalle de 3:2 sans tenir compte des différences de fréquence qui en résultent, alors que d'autres transposent l'intervalle de $54 \mathrm{~Hz}$ (ou $72 \mathrm{~Hz}$ ou $108 \mathrm{~Hz}$ ) sans tenir compte des intervalles qui en résultent, déterminés par le rapport de fréquences.

Pour des exemples de cette exactitude, voir Ellis (1967) ainsi que fig. 3. 
On peut suivre les deux procédés simultanément à l'aide des positions sur la série d'harmoniques, en allant par degrés conjoints (ayant chaque fois la même différence de fréquence et un rapport simple). Par exemple, une série allant graduellement du dix-huitième au douzième harmonique engendre une série de six tons conjoints séparés par la même différence d'une quinte parfaite. En réitérant le dix-huitième et le douzième harmoniques, on circonscrit le principal intervalle de la série. Une fois chiffrés, ces deux tons souvent répétés apparaissent clairement comme des durées maximales, les tons connexes moins souvent utilisés étant nettement moins marqués.

Selon Helmholtz (1863) et Parncutt (1989) - de même que d'autres psychoacousticiens - la série harmonique est perceptible sans autre, mais les auteurs en question se sont généralement limités dans cette matière aux traditions basées sur les instruments. Walcott (1974: 55) analyse et quantifie le chant Chöömij de Mongolie, dans lequel l'interprète ne produit pas seulement la fondamentale de la série mais divise le son en deux séquences de hauteur différente: «l'une, un bourdon nasillard à une hauteur relativement constante, correspond à la fondamentale; l'autre, qui consiste en des sons perçants, sifflants, forme la ligne mélodique sur le bourdon». Ce type de chant a été étudié par d'autres, notamment Smith et al. (1967: 1262ss) au sujet du chant des lamas tibétains, ainsi que Crossley-Holland (1970). David Hykes, en exécutant une version moderne de ce style avec son Harmonic Choir (voir Schaefer 1987: 225) montre que n'importe quel interprète peut adopter cette technique vocale, une fois familiarisé avec elle. Tous les ouvrages mentionnés relèvent l'importance, pour favoriser la clarification de ces harmoniques, du recours à une voix nasillarde et à une fondamentale dont la hauteur atteint $100 \mathrm{~Hz}$ environ. Étant donné que la hauteur du son dans le chant qui nous occupe, en particulier dans la série KE, se situe autour de $108 \mathrm{~Hz}$ pour la tonique, et que la voix des interprètes aborigènes est assez nasillarde, il semble probable qu'ils aient appris la configuration des intervalles à partir de leur perception des sons de cette série.

Les résultats de mon analyse suggèrent que les chanteurs aborigènes, au fur et à mesure qu'ils perfectionnent leur art, se rendent compte, non seulement des rapports d'intervalles simples, comme ils apparaissent dans les harmoniques graves de la série harmonique, mais aussi de la nature de la série elle-même. Les notes le plus souvent répétées s'imposent au premier plan, au niveau de perception de la ligne mélodique, et les tons différentiels se situent à un autre niveau. Les Aborigènes semblent aussi familiers avec les périodes du rythme et le recours à l'ambiguïté tonale pour produire des changements de perception et provoquer le besoin d'une écoute analytique.

Un exemple de ce type d'ambiguïté est fourni par certaines analyses quantitatives plus anciennes (voir Ellis 1964: 300 ss), portant sur des exécutions vocales en solo fortement ornées. Là, le chant semble être en glissement continu, et il est difficile de déterminer une hauteur stable pour un segment quelconque, à partir de laquelle mesurer les intervalles. On n'en est donc pas ici aux fréquences mesurées portant sur des exécutions en groupe, où des durées 
maximales apparaissent clairement, indiquant l'emplacement des notes les plus souvent utilisées.

Pour quelqu'un d'étranger à la tradition, il est tentant d'assumer que le vieil homme qui chante ses invocations sacrées à la pluie pour le chercheur muni de son magnétophone, n'est que le souvenir pathétique d'une tradition vocale jadis florissante. C'est seulement après un réexamen rigoureux plus récent des mesures obtenues que j'ai découvert que l'«intervalle» se définit par la distance du glissement sur n'importe quelle syllabe, par l'espace intertonique rempli de sons plutôt que par l'écart vide entre deux tons individuels. Cela rappelle la recherche de Cogan (1984: 28-35) qui utilise des analyses musicales et spectrales pour montrer comment, dans le chant tantrique, les lamas tibétains se servent de paires de contrastes acoustiques en guise d'oppositions; une de celles-ci implique le glissement, le mouvement oblique et la stase. Une fois reconnu le type de contraste utilisé dans le chant d'Australie centrale, on comprend mieux que l'interprète en question (un chanteur renommé) définit les intervalles par la distance de glissement, et que d'autre part il marque ces glissements par la différence de fréquence. Contrairement aux exécutions chorales discutées plus haut, qui recourent à des intervalles à fréquences différentes basés sur des degrés conjoints de la série harmonique, ces chants en solo utilisent des déplacements difficiles à expliquer en termes d'une quelconque série harmonique, par exemple un glissement de 275 à $248 \mathrm{~Hz}$ (différence de fréquence de $27 \mathrm{~Hz}$ ), suivi par un glissement de 263 à $236 \mathrm{~Hz}$ (nouvelle différence de $27 \mathrm{~Hz}$ ), suivi par un glissement de 252 à $225 \mathrm{~Hz}$ (encore une différence de $27 \mathrm{~Hz}$ ). Ces glissements se déroulent dans une paire globalement marquée d'intervalles extrêmes de 275 à $205 \mathrm{~Hz}$, proche de $4: 3$, et de 248 à $186 \mathrm{~Hz}$ qui est exactement de $4: 3$.

Pour vérifier les détails d'une interprétation quelconque d'un petit chant déterminé en chiffrant l'utilisation globale des tons, il y a avantage à surimposer les tons et les durées transcrits sur une représentation graphique des mesures relevées (voir fig. 1). On obtient ainsi quelques renseignements sur le rapport entre certains tons transcrits et les fréquences mesurées; par là même, on s'aperçoit des difficultés du transcripteur à percevoir ces progressions tonales qui ne ressemblent en rien à ce que l'on rencontre dans la musique européenne.

L'interprétation des tons mesurés lors de l'exécution pose des problèmes considérables, et c'est seulement en mesurant des segments précis d'un petit chant qu'on peut être sûr de la validité de l'interprétation. Nombre d'informations semblent ambiguës, car la descente par degrés conjoints d'intervalles simples le long de la série harmonique signifie aussi l'exécution d'intervalles conjoints ayant la même différence de fréquence. Ce n'est que dans le cas de transpositions qu'on arrive à discerner si l'un ou aucun de ces deux systèmes reste constant. Cela se comprend aisément à l'aide d'un exemple hypothétique.

Une progression à travers la série harmonique de la douzième à la neuvième position à partir d'un ton de $324 \mathrm{~Hz}$ se présente comme suit: 


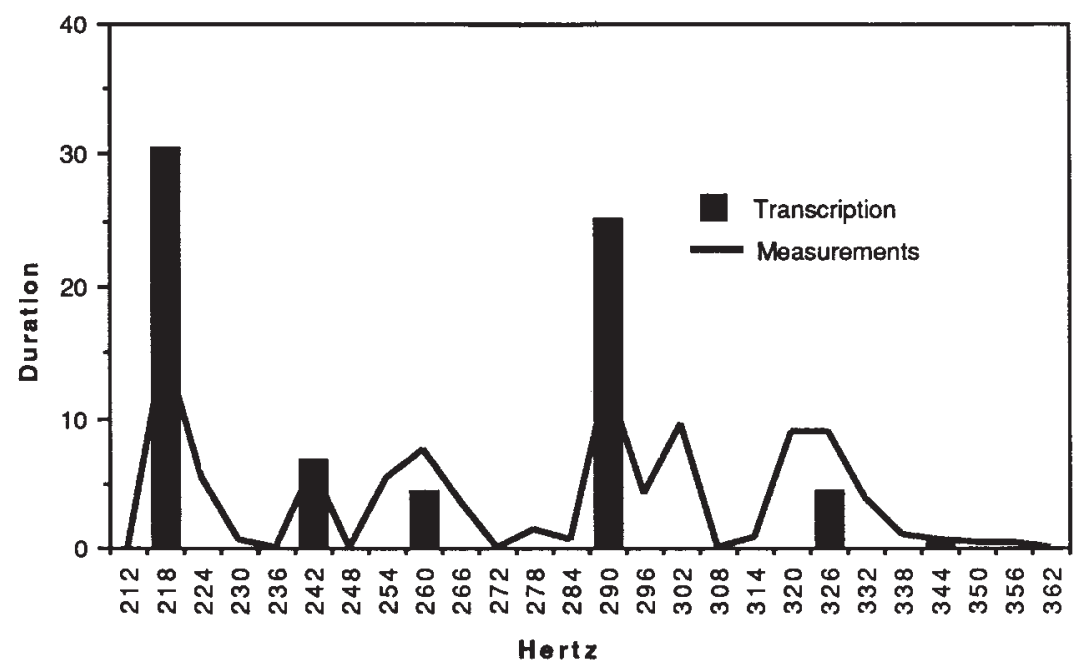

Fig. 1: Comparaison des fréquences mesurées et de la transcription; série KE, petit chant 8(1).

Position sur la série harmonique

Fréquence $(\mathrm{Hz})$

$\begin{array}{rrrr}12 & 11 & 10 & 9 \\ 324 & 297 & 270 & 243\end{array}$

Chacune de ces étapes est séparée à la fois par une série de rapports de fréquences $(12: 11,11: 10,10: 9)$ et par $27 \mathrm{~Hz}$, et les fréquences la plus élevée et la plus basse sont dans un rapport de 4:3. Si cette série est transposée pour maintenir cette progression sur la série harmonique, de même que l'intervalle de 4:3 entre les fréquences au sommet et à la base, les étapes de la série ne seront plus séparées par $27 \mathrm{~Hz}$. Par exemple, si la série commence à $300 \mathrm{~Hz}$, elle se présente comme suit:

Position sur la série harmonique

Fréquence $(\mathrm{Hz})$

$\begin{array}{rrrr}12 & 11 & 10 & 9 \\ 300 & 275 & 250 & 225\end{array}$

Ces intervalles montrent maintenant des écarts de $25 \mathrm{~Hz}$, mais les rapports correspondants et le rapport 4:3 subsistent.

$\mathrm{Si}$, toutefois, la série est transposée par des différences de fréquence égales (dans ce cas les $27 \mathrm{~Hz}$ du premier exemple), comme le suggèrent de nombreux exemples du centre de l'Australie, les fréquences à partir de $300 \mathrm{~Hz}$ sont les suivantes:

Position sur la série harmonique

Fréquence $(\mathrm{Hz})$

$\begin{array}{rrrr}12 & 11 & 10 & 9 \\ 300 & 273 & 246 & 219\end{array}$

On n'a plus ici la même série harmonique (ce serait plutôt une série allant du onzième au huitième), ni exactement l'intervalle de 4:3 qui s'en écarte maintenant de 47 cents (c'est-à-dire presque un quart de ton). L'interprétation des mesures obtenues dépend donc du degré d'exactitude que l'on croit pouvoir 
attribuer à l'intonation dans le chant non accompagné par un instrument définissant la hauteur, ainsi que du degré auquel la transposition semble fondée du point de vue structurel et musical, lorsqu'elle est interprétée à l'aide d'un de ces systèmes ou des deux à la fois.

Le cycle de chants considéré dans ces pages comporte trois versions différentes. Celle que nous appelons la Mélodie 1 est la plus communément utilisée lorsque le chant est exécuté au campement, alors que celle que nous avons définie comme Mélodie 2 est d'usage plus limité, en ce qu'elle accompagne toutes les danses d'ordre cérémoniel. La relation entre ces deux mélodies ressort de la fig. 2: la transition à la note finale aiguë dans la Mélodie 2 est une première preuve qu'il s'agit d'une forme différente. Une répétition de cette mélodie indique que c'est une version cyclique de la Mélodie 1, mais elle en diffère par la note finale ascendante, qui est inhabituelle.

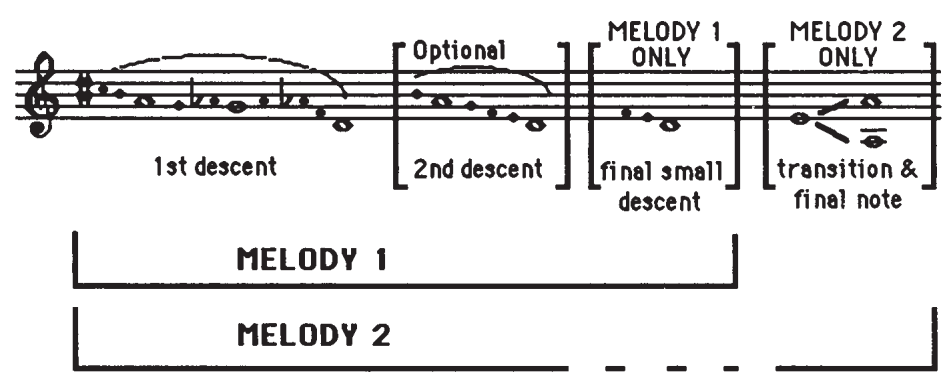

Fig. 2: Modèle de Mélodie 1 et Mélodie 2.

Il existe aussi une troisième mélodie qui n'est exécutée qu'en secret par les hommes, mais comme il n'en existe qu'une seule interprétation analysée et que sa structure est assez complexe pour exiger une analyse séparée, nous la laisserons de côté ici. Chacune des mélodies - 1 et 2 - comprend une ou deux sections mélodiques descendantes.

\section{Mélodie 1}

Dans l'enregistrement de la série KE, réalisé en 1963, le meneur était un chanteur âgé, probablement sexagénaire. Au début, la hauteur du son est instable, et il y a de nombreux désaccords sur l'exécution. Le premier et le second petit chant ont un texte standard $\mathrm{AABB}$, la seconde exécution du premier chant inversant la position du texte par rapport à la mélodie. Dans le second petit chant, de nombreuses corrections énergiques ont lieu, y compris des toussotements bruyants du meneur au moment où les chanteurs se trompent dans la répétition du texte: après avoir chanté les refrains $\mathrm{AABB}$ sur la première descente mélodique, ils auraient dû respirer et passer au registre supérieur pour entamer la nouvelle descente. Toutefois, à ce moment de jointure, ayant réitéré 
le vers A sur la note la plus grave, ils ont dû répéter ce même vers encore deux fois dans le registre supérieur pour réussir la seconde descente.

Avec le passage à la seconde exécution de KE.3, la hauteur et l'ensemble se sont stabilisés, sans doute aidés par le fait que le texte ne comporte qu'une seule séquence descendante. C'est ce petit chant qui favorise la comparaison avec les mesures des fréquences, car celles-ci ont été effectuées séparément pour les vers AA/BBAA qui coïncident avec une descente (voir fig. 3) et pour la section suivant cette jointure qui couvre le refrain BB.

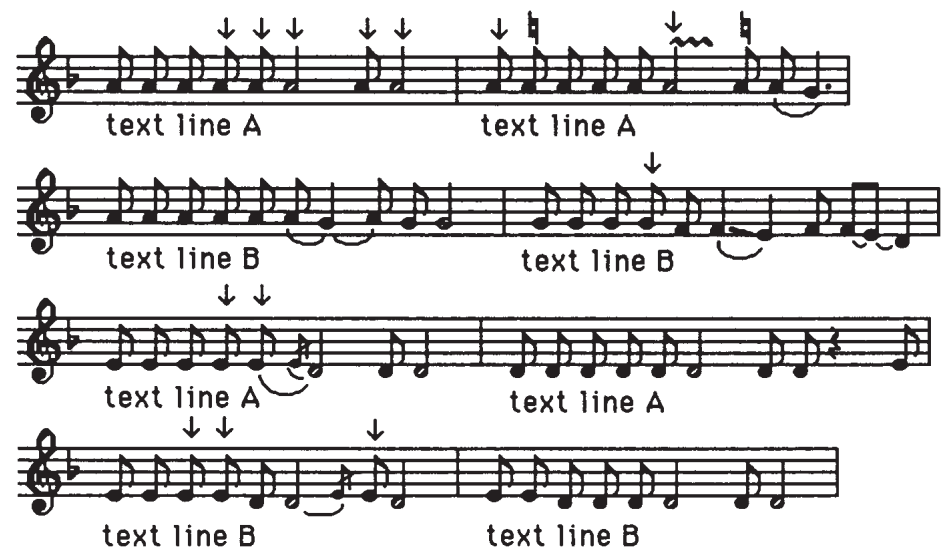

Fig. 3: Petit chant 3(2) de la série KE; texte VI.2.

La représentation graphique des chiffres obtenus montre les durées maximales qui coïncident avec les tons les plus fréquemment utilisés. Dans la fig. 4, on voit une surimposition des deux analyses quantitatives de KE.3(2).

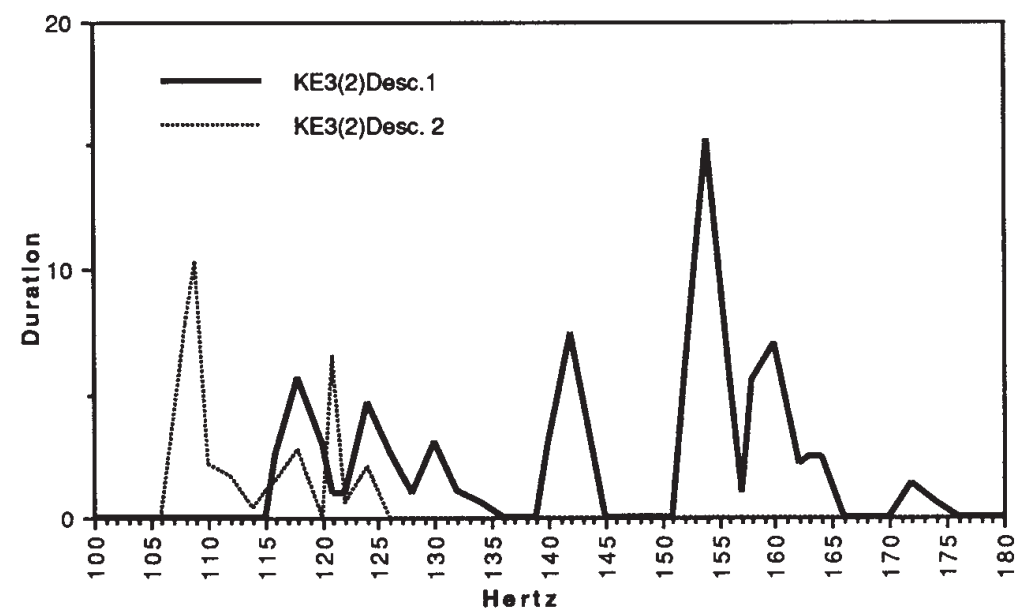

Fig. 4: Principal passage descendant et finale du petit chant 3(2) de la série KE. 
Ces données de KE.3(2) facilitent l'interprétation de KE.2. Tout d'abord, les toniques $(108 / 9 \mathrm{~Hz})$ coïncident dans les deux exemples, ce qui n'est pas le cas des principaux tons aigus. Dans KE.3(2), la principale note aiguë est de $145 \mathrm{~Hz}$, et l'intervalle simple le plus proche par rapport à la tonique se situe ici à $102.6 \mathrm{~Hz}$, ce qui correspond à l'intervalle de 3:2 (quinte parfaite). Dans KE.2, la principale note aiguë atteint $163 \mathrm{~Hz}$, et l'intervalle théorique de 3:2 impliquant la tonique est à $108.6 \mathrm{~Hz}$, ce qui se rapproche de la marge d'erreur des mesures $(+1 \mathrm{~Hz})$. La fig. 4 montre plus clairement ce qui se passe dans le passage descendant vers la tonique et comment les intervalles sont structurés après la jointure avec le texte, marquée ici par une respiration et une élévation vers un ton situé plus haut que la tonique (en réalité l'intervalle est de 10:9).

La section descendante principale de KE.3(3) peut ainsi s'expliquer comme une progression du douzième au neuvième harmonique (c'est-à-dire 4:3) - toutes les fréquences étant arrondies au nombre entier le plus proche, et les notes le plus souvent utilisées imprimées en gras pour chaque ensemble de chiffres (dans tous les cas nous avons montré la mesure la plus proche des fréquences de la série harmonique prévue) :

$\begin{array}{lrrrrr}\begin{array}{l}\text { Position sur la } \\ \text { série harmonique }\end{array} & (13) & 12 & 11 & 10 & 9 \\ \text { Fréquences }(\mathrm{Hz}) & (167) & 154 & 141 & 128 & 115 \\ \text { Mesures }(\mathrm{Hz}) & (172) & \mathbf{1 5 4} & 142 & 130 & \mathbf{1 1 8}\end{array}$

On pourrait aussi l'expliquer selon les différences de fréquences:
Mesures (Hz)
126
154142
(130)
(124) 118
Différence de
$12 \quad 12$
66
fréquence $(\mathrm{Hz})$
(des mesures précédentes)
36

De manière similaire, la brève finale descendante après la jointure avec le texte pourrait être expliquée comme suit:

Position sur la

série harmonique

Fréquences $(\mathrm{Hz})$

Mesures (Hz)
10

121

121
9

109

109

En termes de différence de fréquence, on obtient ceci:

Mesures (Hz)

Différence de

fréquence $(\mathrm{Hz})$ $\begin{array}{lll}124 & 121 & 118\end{array}$ $3 \quad 3$
109

12

Toutes les transcriptions ont été transposées pour que la tonique tombe sur ré. C'est en ayant l'extrême difficulté d'évaluer une transcription exacte que j'ai commencé à mesurer les fréquences des tons, et il est intéressant de relever quelles progressions posent les plus grands problèmes au transcripteur (Barwick). Les fluctuations autour de mi indiquées dans la fig. 2 sont celles qui 
entourent le principal intervalle supérieur de la finale, c'est-à-dire $124 \mathrm{~Hz}$ et $118 \mathrm{~Hz}$ comme inflexions de $121 \mathrm{~Hz}$, ce qui équivaut maintenant à un intervalle de $12 \mathrm{~Hz}$ au-dessus de la tonique.

Étant donné que ces petits chants ont tous la même structure intervallique, une comparaison avec l'analyse quantitative menée pour KE.2 pourrait bien indiquer ce qui se passe dans ce dernier, bien que les quantifications ellesmêmes ne soient pas ici relevées séparément pour chaque mesure descendante.

La première mesure descendante de KE.2 pourrait à nouveau être expliquée comme une progression du douzième au neuvième harmonique (c'est-à-dire 4:3):
Position sur la
série harmonique
Fréquences $(\mathrm{Hz})$
Mesures (Hz)

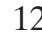

$150 \quad 138$

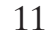

138
10

125

124

\section{9}

113

114

En l'expliquant selon les différences de fréquences, on obtient ceci:

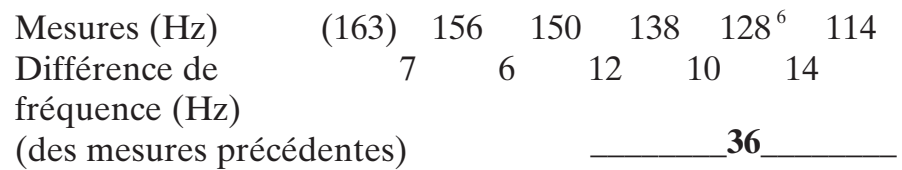

Cette série de différences de fréquences ressemble à la série de la même descente dans KE.3(2).

La seconde séquence descendante de KE.2 commence après une erreur dans le refrain, due à l'exécution erronée d'un vers supplémentaire dans la première mesure descendante; le meneur donne un signal particulièrement vigoureux sur $163 \mathrm{~Hz}$ avant le début de la section suivante qui passe du neuvième au sixième harmonique (intervalle de $3: 2$ ).

\begin{tabular}{|c|c|c|c|c|c|}
\hline $\begin{array}{l}\text { Position sur la } \\
\text { série harmonique }\end{array}$ & & 9 & 8 & 7 & 6 \\
\hline Fréquences (Hz) & & 163 & 144.8 & 126.7 & 108.6 \\
\hline Mesures $(\mathrm{Hz})$ & $(170)$ & 163 & 145 & 128 & 108 \\
\hline
\end{tabular}

On pourrait aussi l'expliquer en termes de différences de fréquences (nous voyons celles-ci dans d'autres exemples avec deux descentes).

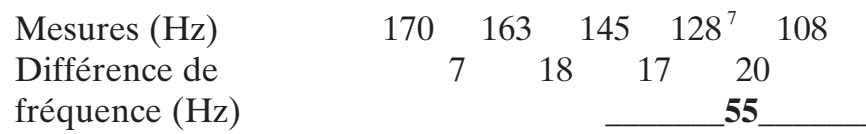

Dans la brève finale descendante de KE.2, il faut voir un recours à la mesure restante grave la plus utilisée n'ayant jusqu'ici pas été sélectionnée ni dans l'une ni dans l'autre série: $120 \mathrm{~Hz}$. Il apparaît ainsi clairement que le passage final

Dans l'exécution, il y a une confusion entre $128 \mathrm{~Hz}$ et $124 \mathrm{~Hz}$; toutes les interprétations suggèrent qu'il faut l'exécuter à $127 \mathrm{~Hz}, 126 \mathrm{~Hz}$ ou $125 \mathrm{~Hz}$.

7 Ici, $127 \mathrm{~Hz}$ auraient donné 7, 18, 18, 19. 
définissant la section inférieure de la mélodie (soit les deux dernières mesures en fig. 3 qui apparaissent toutefois ici après deux descentes mélodiques) est le suivant: $128 \mathrm{~Hz}-120 \mathrm{~Hz}-108 \mathrm{~Hz}$. A nouveau, on a là, soit une étape principale à $12 \mathrm{~Hz}$, soit un intervalle dont le rapport de fréquence se situe à 10:9. La mesure de $128 \mathrm{~Hz}$ est encore une fois irrégulière, car le recours à des intervalles de $6 \mathrm{~Hz}$ est un signal pour les chanteurs qu'une nouvelle séquence descendante va commencer (dans des recherches antérieures j'ai déjà montré ce phénomène); le ton de ce signal aurait donc dû atteindre $126 \mathrm{~Hz}$.

Cette dernière donnée soulève un problème supplémentaire en rapport avec les grandeurs obtenues pour KE.3(2) (voir fig. 4). Cette analyse quantitative a été effectuée à un moment où on était moins informé que maintenant sur la structure des petits chants. Comme l'analyse des fréquences impliquait un travail extrêmement fastidieux, seule la moitié des éléments par série enregistrée était quantifiée, ceux-ci ayant été choisis par échantillonnage aléatoire. Dans KE.3(2), la personne occupée à l'analyse quantitative chercha à découvrir ce qui se passait dans les deux octaves.

Dans la séquence descendante principale, elle établit les hauteurs pour les interprètes chantant dans le registre grave, et dans la brève finale descendante, elle mesura l'octave supérieure. Les chiffres utilisés pour cette section de la fig. 4 ont été divisés par deux afin de correspondre à la transcription. Mais par là même, le petit intervalle du signal de $6 \mathrm{~Hz}$ fut également diminué de moitié, alors qu'il aurait dû rester à $6 \mathrm{~Hz}$ pour toute la tessiture couverte. Les voix aiguës montrent que, soit les chanteurs comptent doubler l'intervalle de la différence de fréquence pour entonner une octave plus haut, soit ils diminuent de moitié s'ils basent leur chant sur des séries harmoniques - la valeur de la fondamentale. Les hauteurs réelles pour cette section finale de KE.3(2) étaient 248 - 242 - 218, ce qui donne une différence de fréquence de $24 \mathrm{~Hz}$ ou un rapport de 10:9.

Un exemple puisé dans la série KK montre le mode de transposition de ces séquences d'intervalles.

La première descente de KK.11 (voir Ellis 1967: 194, 259 pour les graphiques et les transcriptions) se présente probablement comme suit:

$\begin{array}{llllllllllll}\text { Mesures }(\mathrm{Hz}) & 230 & 219 & 208 & \mathbf{1 9 4} & 184 & 171 & 158 & \mathbf{1 4 6} \\ \begin{array}{l}\text { Différence de } \\ \text { fréquence }(\mathrm{Hz})\end{array} & 11 & 11 & 14 & 10 & 13 & 13 & 12\end{array}$

Cela se rapproche de la première descente de l'exemple précédent et peut s'expliquer en termes de séries harmoniques d'une manière presque aussi précise, en considérant qu'on descend du dix-neuvième au douzième harmonique, l'intervalle principal tombant sur 16:12 (4:3). La différence de fréquence de l'intervalle principal est de $48 \mathrm{~Hz}$.

La seconde descente de KK.11 se présente ainsi:

\begin{tabular}{llllllll} 
Mesures $(\mathrm{Hz})$ & 224 & $\mathbf{2 1 2}$ & $\mathbf{1 9 4}$ & 176 & 158 & $\mathbf{1 4 0}$ \\
Différence de & & 12 & 18 & 18 & 18 & 18 \\
fréquence $(\mathrm{Hz})$ & & & & $\mathbf{7 2}$ & & & \\
\cline { 3 - 6 }
\end{tabular}


Ici, le rapport de la différence de fréquence apparaît clairement, et les positions sur la série harmonique qu'il faut pour les exécuter vont du douzième au huitième $(3: 2)$. La différence de fréquence des intervalles principaux (194 -140 , et $212-140$ ) est de $54 \mathrm{~Hz}$ et de $72 \mathrm{~Hz}$.

La finale descendante peut, à nouveau, être déduite de la mesure principale restante dans le registre grave, ainsi que des petits intervalles de signal qui l'entourent:

$\begin{array}{llllll}\text { Mesures }(\mathrm{Hz}) & 158 & & \mathbf{1 5 2} & & \mathbf{1 4 0} \\ \text { Différence de } & & 6 & & 12 & \\ \text { fréquence }(\mathrm{Hz}) & & & & & \end{array}$

Ces hauteurs suggèrent une préférence pour la différence de fréquence. Cette dernière transition est identique à la version exécutée dans l'octave inférieure de KE.3(2), en ce que la différence de fréquence s'accomplit par degrés de $6 \mathrm{~Hz}$, puis de $12 \mathrm{~Hz}$. Toutefois, le rapport d'intervalle de 10:9, lorsqu'il est calculé à partir de $152 \mathrm{~Hz}$, place la tonique à $136,8 \mathrm{~Hz}$ : voilà un léger écart par rapport à ce qu'indiquent les autres intervalles au sujet de l'exactitude de l'intonation du chanteur (une erreur de 40 cents seulement, mais de zéro pour l'interprétation de la différence de fréquence sur la seconde mesure descendante et la finale). Le rapport actuel est de 13:12. Une analyse de KK, qui ne laisse subsister aucun doute quant à la hauteur de cette brève finale descendante, a été effectuée pour KK.2 (voir Ellis 1967: 189). Voici sa séquence finale: $176 \mathrm{~Hz}-\mathbf{1 7 0 ~ H z}-158 \mathrm{~Hz}$. Dans cet exemple, la différence de fréquence reste la même, mais le rapport d'intervalle de 10:9 indique un écart de 56 cents, le rapport nécessaire de l'intervalle mesuré est de 14:13. Ainsi y at-il constamment une différence de fréquence, mais avec chaque transposition, le rapport de fréquences change.

A ce stade, on voit clairement que, bien que les structures soient ambiguës, seul un des systèmes est parfaitement approprié s'il y a une transposition substantielle. Ce qui semble se passer, c'est que la première mesure descendante commence par un petit intervalle $(6 \mathrm{~Hz})$, situé directement au-dessus de la note principale de la série (probablement un repère du groupe pour faciliter le chant d'ensemble), et l'accent est mis sur les degrés de $12 \mathrm{~Hz}$ dans toute la gamme descendante. Dans la seconde mesure descendante, l'accent est placé sur les degrés plus larges de $18 \mathrm{~Hz}$, encore une fois introduits par un petit intervalle, et cette fois-ci l'accent est mis à la fois sur l'intervalle de 3:2 et sur la différence de fréquence de $54 \mathrm{~Hz}$, même dans les segments transposés. Dans KK.11, aussi bien l'intervalle de $54 \mathrm{~Hz}$ que l'intervalle de $3: 2$, toujours au-dessus de la tonique, apparaissent comme des durées clairement marquées et séparées, tandis que dans la série $\mathrm{KE}$, la hauteur de la tonique permet aux deux de coïncider. Dans aucun des exemples de KE, l'intervalle principal de la première descente n'est exactement de 4:3 (mais il s'en rapproche suffisammment), et la même observation vaut pour l'exemple de KK ; mais dans les deux cas se rapportant à KE, une différence d'exactement $36 \mathrm{~Hz}$ sépare les deux notes principales: 154 - 118 et 150 - 114, alors que dans KK, 
c'est $48 \mathrm{~Hz}$, et le lien avec les $36 \mathrm{~Hz}$ ressort de l'intervalle de $72 \mathrm{~Hz}$ dans la seconde mesure descendante.

La jointure avec le texte contient deux informations importantes sur la hauteur. Le tableau suivant donne les fréquences de la tessiture principale des sections déterminées par la jointure du texte et de la mélodie pour trois éléments, les notes les plus fréquemment utilisées étant chiffrées.

$\begin{array}{llll} & 1^{\text {re }} \text { descente } & 2^{\text {e }} \text { descente } & 3^{\mathrm{e}} \text { descente } \\ \text { KE.2 } & 150-114 \mathrm{~Hz}(36) & 173-108(55) & 120-108(12) \\ \text { KE.3(2) } & 154-118 \mathrm{~Hz}(36) & \text { aucun } & 121-109(12) \\ \text { KK.11 } & 194-146 \mathrm{~Hz}(48) & 194-140(54) & 152-140(12)\end{array}$

Les rapports de fréquence correspondants sont:

$\begin{array}{llll} & 1^{\text {re }} \text { descente } & 2^{\text {e }} \text { descente } & 3^{\mathrm{e}} \text { descente } \\ \text { KE.2 } & 4: 3 & 3: 2 & 10: 9 \\ \text { KE.3(2) } & 4: 3 & - & 10: 9 \\ \text { KK.11 } & 4: 3 & 11: 8 & 13: 12\end{array}$

\section{Mélodie 2}

Dans l'analyse quantitative de la seconde mélodie considérée dans ces pages, il y a un exemple avec une ouverture descendante partielle, qui permet de voir le déroulement d'autres exécutions. KE.18(2) comporte une ouverture qui se fait parfois dans les petits chants de la Mélodie 2, en descendant à travers à peu près la moitié de la tessiture employée (les hauteurs relevées indiquent qu'elle s'étend sur plus d'une tierce majeure: 423 cents) dans les deux premiers vers. Les fréquences mesurées peuvent aisément être interprétées en les situant entre le dix-huitième et (potentiellement) le douzième harmonique $(3: 2)$.

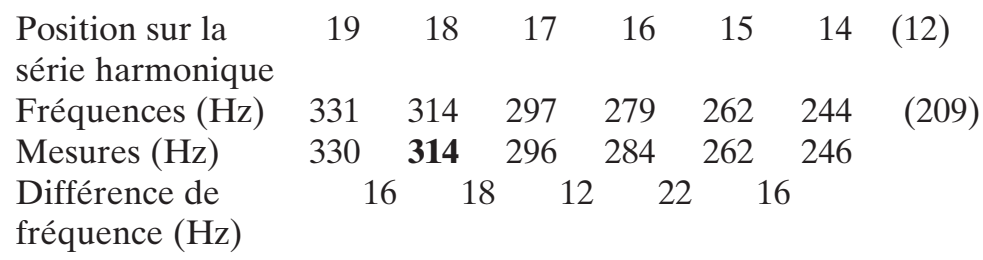

Un bon exemple des processus s'appliquant à la Mélodie 2 ressort des petits chants 16(1) et 16(2) dont l'intérêt ne réside pas seulement dans la présentation conjointe du seul texte utilisé dans l'exécution, mais aussi dans le fait que 16(1) étant la première utilisation de la Mélodie 2 dans la série KE, il y a quelques erreurs intéressantes dans l'exécution (voir fig. 5).

Dans la première présentation de la Mélodie 2 dans KE, il y a une confusion dans l'exécution qui semble tenir à un doute concernant la séquence à suivre dans chacune des descentes mélodiques, et à la question de savoir si la première d'entre elles doit être basée sur la série harmonique précédemment utilisée 

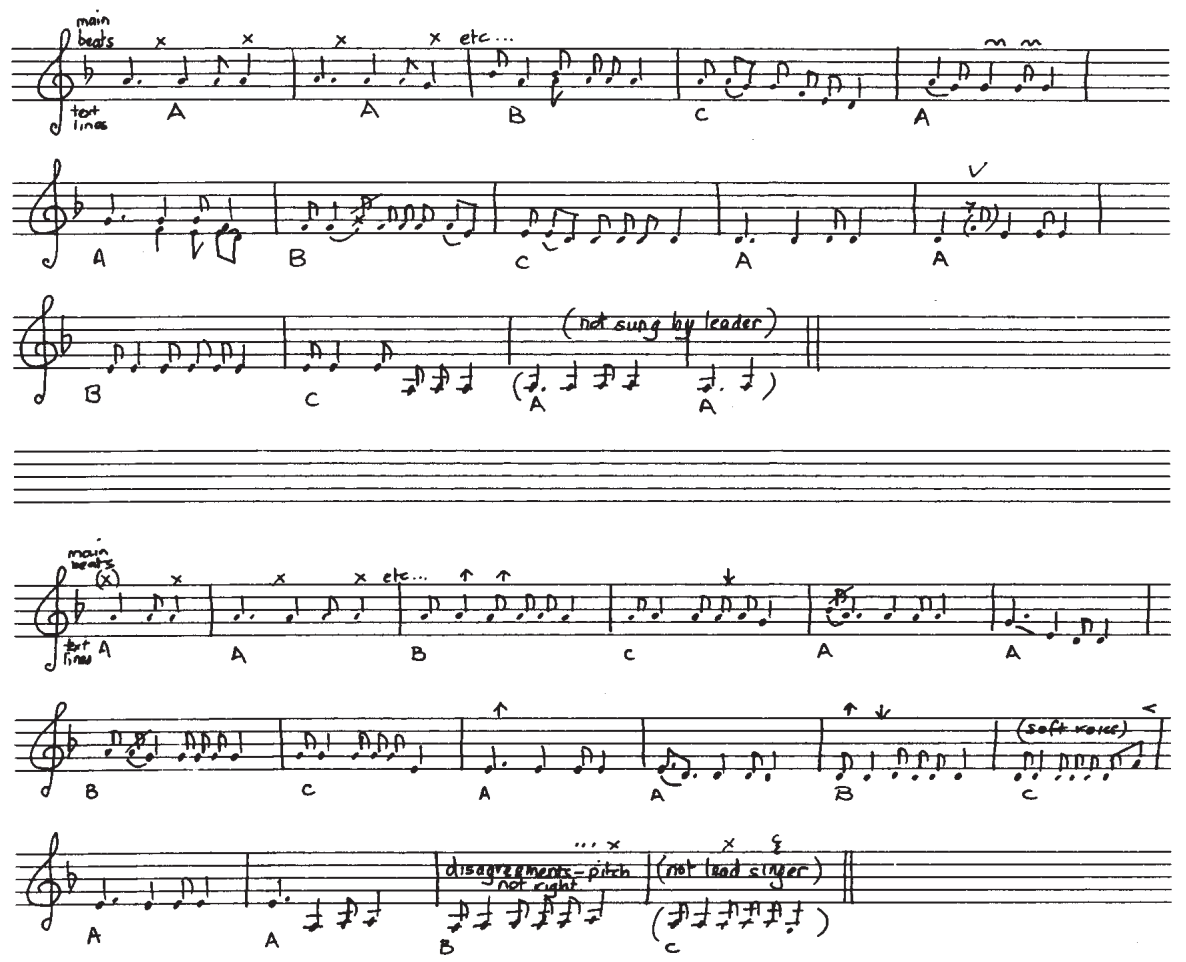

Fig. 5: Petits chants 16(1) et 16(2) de la série KE; texte III.2b.

ou sur les différences de fréquence. Dans la première mesure descendante de KE.16(1), la série est à nouveau ambiguë, car les différences de fréquence relevées de 108 Hz (c'est-à-dire deux fois la grandeur de l'intervalle récurrent de $54 \mathrm{~Hz}$ dans l'exemple de la Mélodie 1 transcrit une octave plus bas) est le plus souvent situé entre la note principale supérieure et la tonique, mais la note finale de cette séquence descendante $(214 \mathrm{~Hz})$ n'est pas la tonique; $207 \mathrm{~Hz}$ est la valeur plus large de la durée.

\begin{tabular}{|c|c|c|c|c|c|c|c|}
\hline $\begin{array}{l}\text { Position sur la } \\
\text { série harmonique }\end{array}$ & 18 & 17 & 16 & 15 & 14 & 13 & 12 \\
\hline Fréquences (Hz) & 321 & 303 & 285 & - & - & 232 & 214 \\
\hline Mesures (Hz) & 322 & 306 & 284 & - & - & 230 & 214 \\
\hline $\begin{array}{l}\text { Différence de } \\
\text { fréquence }(\mathrm{Hz})\end{array}$ & 16 & & & 108 & & & \\
\hline
\end{tabular}

La confusion de hauteur est la plus notable dans la transcription au début de la seconde section, qui suit directement la première jointure principale. La deuxième note principale semble avoir été interprétée par quelques chanteurs 
selon une série harmonique différente de celle de la première mesure descendante. De plus, quelques chanteurs se sont trompés sur la jointure véritable. Le groupe principal a chanté les vers $\mathrm{AABC}$ avec le premier passage descendant dans le vers $C$, et un passage ascendant pour commencer le nouveau vers $A$; tandis que d'autres ont interprété les vers initiaux AA comme la brève ouverture descendante déjà discutée plus haut pour le petit chant 18(2), puis chanté le texte intégral dans la forme (AA)BCAA, avec un passage descendant dans le vers A final. Cela provoque non seulement une confusion dans les tons utilisés (pour chanter en «harmonie») mais dans le positionnement de la jointure. A la fin du petit chant, après de nombreux petits intervalles fortement accentués par le meneur pour guider les chanteurs vers la hauteur correcte, le chant se termine convenablement.

La seconde mesure descendante exécutée par le groupe principal des chanteurs semble suivre fondamentalement la même série harmonique que la première, encore une fois avec plusieurs positions possibles mais inutilisées. Ici, l'intervalle est plus régulier et plus proche des intervalles de la Mélodie 1, et la rétrogradation pourrait être intentionnelle; l'intervalle majeur est identique à celui de la première descente, bien qu'il se déplace de $7 \mathrm{~Hz}$.

\begin{tabular}{|c|c|c|c|c|c|c|c|c|}
\hline $\begin{array}{l}\text { Position sur la } \\
\text { série harmonique }\end{array}$ & & 18 & (17) & 16 & 15 & 14 & (13) & 12 \\
\hline Fréquences $(\mathrm{Hz})$ & & 315 & (298) & 280 & 263 & 245 & (228) & 210 \\
\hline Mesures $(\mathrm{Hz})$ & 322 & 315 & & 279 & 260 & 242 & & 207 \\
\hline $\begin{array}{l}\text { Différence de } \\
\text { fréquence }(\mathrm{Hz})\end{array}$ & & & 36 & 19 & & 18 & 35 & \\
\hline
\end{tabular}

Ici, le passage à la note au-dessus de la tonique est faux par comparaison avec d'autres exécutions de la Mélodie 2, mais il n'en concorde pas moins avec la brève finale descendante de la Mélodie 1. La hauteur normale de cette note dans la Mélodie 2 diffère de celle de la Mélodie 1. Cette dernière, comme nous l'avons montré pour KE, fut interprétée, soit comme $12 \mathrm{~Hz}$ (ou $24 \mathrm{~Hz}$ à l'octave supérieure), soit comme l'intervalle de 10:9. La hauteur de cette note dans le petit chant KE.16(1) est de $230 \mathrm{~Hz}$ (c'est-à-dire $23 \mathrm{~Hz}$ ou 10:9 au-dessus de la tonique), mais elle se situe généralement vers $235 \mathrm{~Hz}$. Dans la Mélodie 2, en raison de l'intervalle de 4:3 au-dessus de cette note et de 3:2 au-dessous d'elle, pour la finale correcte, les chanteurs auraient dû reprendre la note principale aiguë de $315 \mathrm{~Hz}$, mais en employant $230 \mathrm{~Hz}$ comme tremplin pour ce saut, ils ne parviennent qu'à $307 \mathrm{~Hz}$ (ou 153,3 Hz à la place des $164 \mathrm{~Hz}$ exécutés).

Il y a beaucoup de confusion sur la façon d'atteindre cette hauteur finale, et c'est seulement dans des segments ultérieurs qu'une structure régulière est établie. Ici cependant, il semble que la confusion provienne du fait que les chanteurs ont appliqué erronément l'intervalle de 10:9 $(24 \mathrm{~Hz})$.

Dans KE.16(2), soit la seconde exécution d'un texte identique à celui de 16(1), il y a une confusion supplémentaire. Tout d'abord, les vers sont inversés, et quelques membres du groupe opèrent la jointure au même endroit que dans 16(1), c'est-à-dire après l'exécution des vers AA/BCAA. La hauteur initiale est 
à nouveau instable, la première descente commençant à $338 \mathrm{~Hz}$ et procédant du dix-huitième au seizième harmonique avant de terminer ce segment. Une fois arrivé à la principale section descendante, la transition du dix-huitième au douzième harmonique est identique à 16(1), mais, cette fois-ci, sur un ordre différent des vers (BCAA). Cette finale de 16(2), dont le transcripteur affirme qu'elle est impossible à déterminer, est plus proche de la formule mélodique usuelle. Pour comprendre la nature ambiguë de la construction des intervalles de cette finale, il faut des explications théoriques supplémentaires.

Lorsqu'une octave est divisée en deux par l'intervalle de 3:2 au-dessus de la note la plus grave, cette division se fait en deux intervalles à la différence de fréquence égale. Ainsi l'intervalle de fréquence inscrit dans l'octave de 314 à $157 \mathrm{~Hz}$ est de $157 \mathrm{~Hz}$; la hauteur de l'intervalle de 3:2 au-dessus de 157 est de 235,5 Hz, et celui de 4:3 au-dessus atteint $314 \mathrm{~Hz}$. Chacun de ces intervalles est séparé par 78,5 Hz. Toutefois, si l'on considère l'octave inscrite dans cette gamme descendante dans les exemples de la Mélodie 2 comme étant divisée par la tonique - 207 -, alors la division de l'octave par 4:3 au-dessous de $207 \mathrm{~Hz}$ (à $155 \mathrm{~Hz}$ ) et par 3:2 au-dessus de lui $(311 \mathrm{~Hz})$ donne des intervalles fréquentiels qui sont eux-mêmes dans un rapport de 3:2 de l'ensemble et de 3:2 l'un par rapport à l'autre. Il faut avoir la hauteur de la tonique et de l'octave supérieure et inférieure de la note finale dans la gamme précise et correcte pour arriver à l'idéal qui préserve l'ambiguïté entre la différence de fréquence des intervalles majeurs et l'interprétation correspondante de ces mêmes intervalles selon le rapport de fréquence. Le petit chant 16(2) s'en rapproche presque; en voici les mesures:

«Octave» d'encadrement $(\mathrm{Hz})$

Différence de fréquence $(\mathrm{Hz})$
154 314

La moitié de cette différence de fréquence (c'est-à-dire 80) donne la fréquence de la note principale au-dessus de la tonique, d'où l'on saute aux octaves extérieures:

$$
314 \mathrm{~Hz}-80 \mathrm{~Hz}=234 \mathrm{~Hz}
$$

et dans KE.16(2) la hauteur atteignait $235 \mathrm{~Hz}$.

Un tiers de la différence de fréquence sur l'octave chantée est 53.3, valeur qui rappelle les $54 \mathrm{~Hz}$ apparaissant fréquemment dans les exemples de la Mélodie 1 et la différence de fréquence de $108 \mathrm{~Hz}$ rencontrée dans les exemples de la Mélodie 2. En divisant l'échelle en deux, puis en trois sections à partir du ton aigu, on obtient:

$$
314-53.3 \times 2(106.6)=207
$$

ce qui est la hauteur de la tonique dans la plupart de ces chants de Mélodie 2; et

$$
314-53.3 \times 3=154.1
$$

qui est la hauteur la plus basse obtenue pour 16(2).

Si on la considère selon les positions sur la série harmonique, la série 4:3:2 à partir de la note la plus aiguë donne la hauteur centrale de la note de transition, 


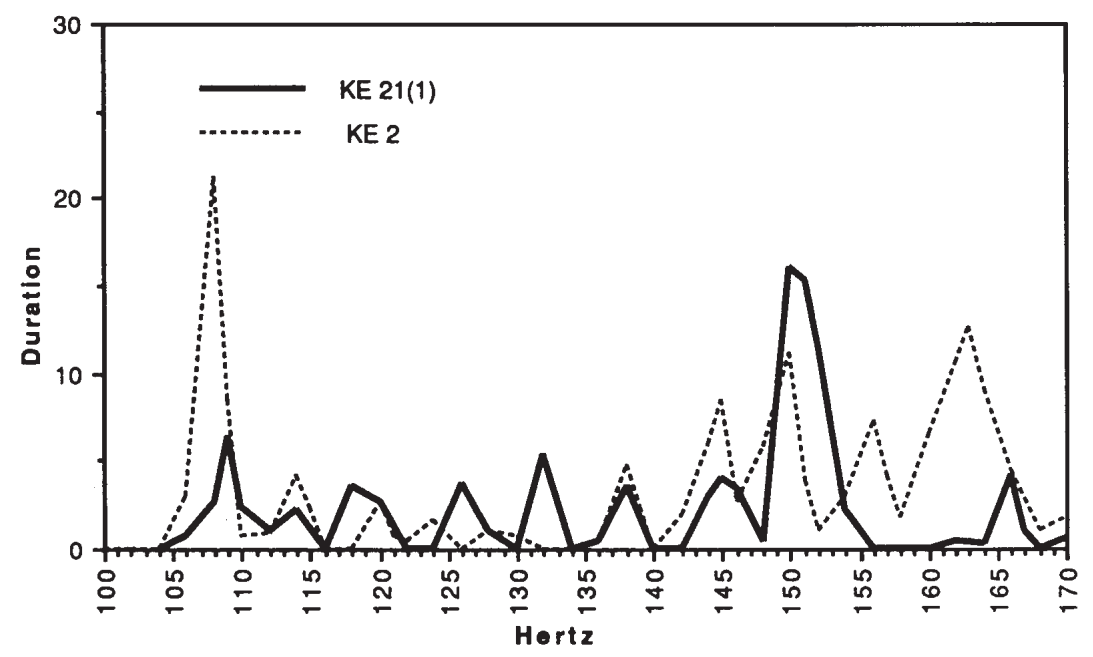

Fig. 6: Surimposition des petits chants 2 et 21(1) de la série KE.

alors que la rétrogradation de l'intervalle 2:3:4 à partir de la note la plus aiguë donne la hauteur de la tonique. Il y a divers traits, dont ce dernier, qui suggèrent que la Mélodie 2, alors qu'elle maintient constantes les positions sur la série harmonique, comporte une manipulation des rétrogradations. Un autre exemple est la séparation mélodique du refrain AA comme un bref passage descendant au-dessous de la note aiguë, alors que le passage ascendant à la note transitoire au-dessus de la tonique est de manière similaire exécuté sur un refrain. La fig. 6 surimpose KE.2 (Mélodie 1) et KE.21(1) (Mélodie 2); et le graphique montre également une rétrogradation supplémentaire: la durée de la tonique de KE.2 est identique à celle de la note principale aiguë de KE.21(1), ce qui suggère que toute la Mélodie 2 pourrait être structurée comme une inversion d'intervalles de la Mélodie 1, les deux notes supérieures de cette dernière et leur passage descendant à la tonique étant reflétées dans la tonique et la note transitoire de la Mélodie 2, les deux s'élevant à la note principale aiguë, et il agit pour cela probablement comme la tonique. L'ambiguïté de la division de l'octave extérieure dans les versions de la Mélodie 2 qui utilisent la forme à la fois inférieure et supérieure de la note finale peut donc être considérée comme l'ambiguité entre la tonique considérée, soit comme la note la plus aiguë, soit comme la note plus basse qui est normalement la tonique. 


\section{Conclusion}

Notre analyse suggère que les chanteurs d'Australie centrale ont une capacité extraordinaire d'exécuter une division exacte et précise d'intervalles larges; de maintenir la tonique dans une position où la différence de fréquence demeure constante; de tenir compte des signaux du meneur pour arriver à des gammes descendantes correctes; de connaître le texte et sa jointure avec la mélodie; d'appliquer toutes ces informations aux petits chants plutôt compacts (qui durent rarement plus de trente secondes) tout comme aux longues séries de chants dont l'interprétation intégrale peut s'étaler sur plusieurs jours. Tout cela démontre que les interprètes aborigènes sont des musiciens particulièrement habiles, et nous considérons notre travail avec eux comme un véritable privilège. Dommage que les autorités en matière d'instruction publique, qui interviennent dans la région où ces gens continuent à vivre et à pratiquer leurs chants, ne partagent pas notre grand respect pour leur art vocal et leur capacité de maintenir la pratique de chants dont la forme actuelle a été façonnée à travers d'innombrables générations d'interprètes.

\section{Bibliographie}

BARWICK Linda

1989 «Creative (ir)regularities: the intermeshing of text and melody in performance of Central Australian song». Australian Aboriginal Studies 1: 12-28.

1990 «Central Australian women's ritual music: knowing through analysis versus knowing through performance». Yearbook for Traditional Music 22: 60-79.

COGAN Robert

1984 New Images of Musical Sound. Cambridge, Ma.: Harvard University Press.

CROSSLEY-HOLLAND Peter

1970 «rGya-gLing hymns of the Karma-Kagyu: the rhythmitonal architecture of some Tibetan instrumental airs». Selected Reports in Ethnomusicology 1(3): 80-114.

ELLIS Catherine $\mathrm{J}$.

1964 Aboriginal Music Making. A Study of Central Australian Music. Adelaide: Libraries Board of South Australia.

1965 «Pre-instrumental scales». Ethnomusicology 9(2): 126-44.

1967 «The Pitjantjatjara kangaroo song from Karlga». Miscellanea Musicologica: Adelaide Studies in Musicology 2: 170-262.

1984 «Time consciousness of Aboriginal performers». In: Problems and Solutions. Occasional Essays in Musicology Presented to Alice M. Moyle. Éd. J.C. Kassler \& J. Stubington. Sydney: Hale \& Iremonger, p. 149-85.

1985 Aboriginal Music: Education for Living. Cross-Cultural Experiences from South Australia. St. Lucia: University of Queensland Press (reissued in paperback 1989).

s.d. «Transposition of South Australian Aboriginal songs: frequency ratio or frequency difference?» In: Festschrift for Prof. Kuckertz. Éd. R. Schumacher. Berlin: [sous presse].

ELLIS C.J., A.M. ELLIS, M. TUR \& A. McCARDELL

1978 «Classification of sounds in Pitjantjatjara-speaking areas. In: Australian Aboriginal Concepts. Éd. L. Hiatt. Canberra: Australian Institute of Aboriginal Studies, pp. 68-79. 
HELMHOLTZ Hermann von

1863 On the Sensation of Tone as a Physiological Basis for the Theory of Music. English Translation by A.J. Ellis 1885. With a new introduction by Margeneau. New York: Dover Publications, 1954.

JONES Trevor A.

1980 «Australian Aboriginal music». In: New Groves Dictionary of Music and Musicians, vol. 1. Ed. S. Sadie. London: McMillan, pp. 711-13.

McCARDELL A.

1976 Rhythm and Melody in Australian Aboriginal Songs of the Western Desert, vol. 1. Unpublished PhD Thesis, University of Western Australia.

MOYLE Richard

1979 Songs of the Pintupi. Musical Life in a Central Australian Society. Canberra: Australian Institute of Aboriginal Studies.

1984 «Jumping to conclusions». In: Problems and Solutions. Occasional Essays in Musicology Presented to Alice M. Moyle. Éd. J.C. Kassler \& J. Stubington. Sydney: Hale \& Iremonger, pp. 51-58.

PARNCUTT R.

1989 Harmony. A Psycho-acoustical Approach. Berlin: Springer-Verlag.

\section{SCHAEFER J.}

1987 New Sounds. New York: Harper \& Row.

SMITH H., K.N. STEVENS \& R.S. TOMKINSON

1967 «On an unusual mode of chanting by certain Tibetan lamas». Journal of the Acoustical Society of America 61: 1262.

STREHLOW T.G.H.

1971 Songs of Central Australia. Sydney: Angus \& Robertson.

TUNSTILL Guy

1985 Music and language, and other aspects of Pitjantjatjara song. Unpublished paper, University of New England, Armindale.

WALCOTT Ronald

1974 «The Chöömij of Mongolia: a spectral analysis of overtone singing». Selected Reports in Ethnomusicology 2(1): 55-60.

WILD Stephen A.

1984 «Warlbiri music and culture: meaning in a Central Australian song series». In: Problems and Solutions. Occasional Essays in Musicology Presented to Alice M. Moyle. Éd. J.C. Kassler \& J. Stubington. Sydney: Hale \& Iremonger, pp. 186-203. 